Ignez Salas Martins 1

Gustavo Velásquez-Meléndez 1

Ana Maria Cervato 1

\footnotetext{
1 Faculdade de Saúde Pública, Departamento de Nutrição, Universidade de São Paulo. Av. Dr. Arnaldo 715 , São Paulo, SP

01246-904, Brasil.
}

\section{Estado nutricional de grupamentos sociais da área metropolitana de São Paulo, Brasil}

\author{
N utritional status of social groups in greater \\ metropolitan São Paulo, Brazil
}

Abstract This paper focuses on prevalence of nutritional status (Body Mass Index, BMI) in social groups in Greater Metropolitan São Paulo, in Southeastern Brazil. The population was stratified in four socioeconomic groups. Prevalence of malnutrition (BMI $<18.5 \mathrm{~kg} / \mathrm{m} 2)$, low body mass (BMI <20.0kg/m2), and overweight (BMI $>25.0 \mathrm{~kg} / \mathrm{m} 2)$ and obesity $(B M I \geq 30.0 \mathrm{~kg} / \mathrm{m} 2)$ were calculated. Prevalence of malnutrition was 3.9\% in men and $6.2 \%$ in women. Prevalence of overweight ranged from $27.5 \%$ to $34.1 \%$ in men and from $25.8 \%$ to $43.6 \%$ in women. Obesity ranged from $2.5 \%$ to $11.1 \%$ in men and from $7.1 \%$ to 20.5 in women. Prevalence of overweight and obesity was greater among women than men $(p<0.01)$. In relation to excess weight $(B M I>25.0$ $\mathrm{kgm} / 2$ ), the study showed that prevalence in men was $43,51,35$, and $30 \%$ for strata I, II, III, and IV, respectivel y. Among women, preval ence was 12, 61, 55 and 46\% for strata I, II , III, and IV, respectively. In women there were an abrupt increase in excess weight just before 40 years of age. A high percentage of overweight and obesity was observed in all population groups. Key words Nutritional Status; Body Mass Index; Anthropometry; Epidemiology

Resumo O presente trabal ho tem como objetivo caracterizar a prevalência de baixo peso e obesi dade em grupamentos (estratos) sociais da Área Metropolitana de São Paulo, Brasil. O estado nutricional foi definido pelo Índice de Massa Corporal (IMC). As prevalências de desnutrição energético-protéica foi de 3,9\% nos homens e 6,2\% nas mulheres. O sobrepeso variou de $27,5 \%$ a $34,0 \%$, nos homens e de $25,8 \%$ a $43,6 \%$, nas mulheres. A obesidade, nos homens, variou de $2,5 \%$ a $11,1 \%$; nas mulheres de $7,1 \%$ a $28,5 \%$. Nestas o excesso de peso apresentou aumento brusco após os 40 anos de idade, com diferenciais estatisticamente significantes ( $p \varangle 0,001)$, entre os se xos. Nos estratos sociais as prevalências de I MC <20,0 variaram entre 8 e $24 \%$. Entre as mul heres, entre $10 \%$ (estrato I) e $26 \%$ (estrato IV). As prevalências de sobrepeso e obesi dade entre os homens foram deaproximadamente 43, 51, 35 e 30\% para as classes I, II, III e IV, respectivamente. Entre as mulheres, os percentuais foram de mais ou menos 12, 61, 55 e $46 \%$ para os estratos I, II, III eIV, respectivamente. O sobrepeso e obesi dade encontram-se em alta prevalência em toda população.

Palavras-chave Estado Nutricional; Índice de Massa Corporal; Antropometria; Epidemiologia 
Introdução

A obesidade apresenta-se como doença da modernidade, resultante do desenvolvimento tecnológico que proporcionou menor escassez de alimentos, aliado às mudanças observadas na composição das dietas com o aumento no consumo de gorduras, açúcar, alimentos refinados e redução na ingestão de carboidratos complexos e fibras-tendência denominada de "transição nutricional". A vida sedentária, outra consequência da modernidade, juntamente com a "transição nutricional" compõem os principais fatores etiológicos da obesidade (Monteiro et al., 1995).

No Brasil o problema da obesidade vem se agravando nas últimas décadas. A comparação entre dois estudos de abrangência nacional, o primeiro em 1974 e o segundo em 1989, demonstra aumento expressivo da obesidade acompanhada de diminuição do número de casos de desnutrição energético-protéica, em adultos entre 25 a 64 anos de idade. A "transição nutricional" e a redução na proporção de famílias muito pobres teriam sido, entre outros, fatores determinantes do aumento da densidade energética das dietas brasileiras e da disponibilidade de alimentos (Mondini \& Monteiro, 1995).

Por outro lado, o presente estudo, realizado na área metropolitana de São Paulo, procurou caracterizar perfis de estado nutricional de grupamentos típicos da sociedade brasileira definidos por critérios sócio-econômicos e pelo modo de inserção na vida urbana. Faz parte de um projeto sobre fatores de risco para doenças cardiovasculares ateroscleróticas desenvolvido em um Município da Grande São Paulo (Martins et al., 1993). Foram definidas “áreas de estudo" que se constituíam em pequenas áreas geográficas, inseridas em diferentes pontos do meio urbano.

Assim, conseguiu-se apreender alguns aspectos relacionados à grande diversidade de qualidades de vida que compõem a grande São Paulo. Neste sentido, pressupõe-se que a desnutrição e a obesidade se apresentam como problemas de Saúde Pública na região, na medida em que são situações fisiológicas determinadas por condições sócio-econômicas.

Assim, é objetivo deste trabalho caracterizar estados nutricionais - obesidade e baixo peso - nos grupamentos sociais definidos nessa pesquisa.
Material e métodos

Populacão e áreas de estudo

A localidade estudada foi o Município de Cotia, situado a oeste da Área Metropolitana de São Paulo, entre 1990 e 1991. A metodologia adotada baseou-se na delimitação de pequenos núcleos urbanos, denominados "áreas de estudo", através de critérios sócio-econômicos e geográfico (Castells, 1971; Breilh \& Granda, 1980; Laurell, 1982; Lombardi et al., 1988; Singer, 1981). Cada uma dessas áreas era, na medida do possível, homogênea, representando modos típicos de inserção na sociedade e, entre as cinco definidas, pode-se obter um gradiente de qualidade de vida.

A delimitação das "áreas de estudo" teve duas finalidades: a de permitir a definição de áreas homogêneas do ponto de vista sócioeconômico e a de facilitar o entrosamento da equipe com as associações e serviços locais de saúde, que passariam a deter informações sobre o estado de saúde da população envolvida e a se responsabilizar pelo encaminhamento à atenção clínico-educativa pertinente.

As "áreas de estudo" foram definidas a partir dos setores censitários do Município, nos quais foram feitos levantamentos das associações comunitárias, dos serviços de saúde e dos respectivos fluxos de clientela.

Foram definidas cinco áreas, Figura 1, localizadas em diferentes pontos geográficos do Município, cujos perfis no que tange à inserção na vida urbana podem ser assim delineados:

- Área 1: formada por famílias assentadas na região há várias gerações e por migrantes recentes, principamente da Bahia. É constituída de pequenos comerciantes e trabal hadores não qualificados. Nesta primeira "área de estudo" foi realizado um estudo piloto, no qual foram testados métodos e técnicas a serem empregados na pesquisa.

- Área 2: limítrofe com um bairro de população de alta renda e abrigando, principalmente, profissionais liberais, operários qualificados, médios empresários e comerciantes.

- Área 3: formada no início da década de 70 por migrantes provenientes do interior de São Paulo e de outros estados, principalmente de Minas Gerais. É formada, basi camente, por trabalhadores não qualificados e pequenos comerciantes.

- Área 4: próxima ao centro do Município, formada principalmente por trabalhadores do setor terciário, comerciantes e pequenos empresários. 
- Área 5: centro do Município, formada por profissionais liberais, comerciantes e trabalhadores de setor terciário, principalmente funcionários públicos.

A amostra utilizada foi obtida a partir de critérios que possibilitariam a realização de inquéritos clínico-bioquímico e alimentar, fixando-se um coeficiente de variação/ ano superior a 5\% para qualquer estimativa a ser estudada. Nas áreas estabelecidas, o número de indivíduos amostrados foi de 1041, tendo sido estratificados em cinco estratos, tomando-se como base as categorias renda, ocupação e escolaridade, com as seguintes características:

\section{Estratos sociais}

- Estrato 1: formada por empresários ou comerciantes proprietários, com mais de cinco empregados e profissionais de nível universitário;

- Estrato 2: formada por pequenos empresários ou comerciantes com menos de cinco empregados, com nível médio de escolaridade e rendimentos mensais maiores de cinco salários mínimos;

- Estrato 3: formada por empregados assalariados com conhecimento de ofício, de nível primário ou médio de escolaridade;

- Estrato 4: formada de sub-empregados, sem conhecimento de ofício, pequenos proprietários com rendimentos abaixo de cinco salários mínimos e nível de escolaridade correspondente ao primário incompleto ou analfabeto.

\section{Estado nutricional}

O estado nutricional foi medido pelo "Índice de Massa Corporal" (peso/ altura2), tendo sido definidas as seguintes categorias (Bray, 1985; Ferro-Luzzi et al., 1992; James et al., 1988; Coitinho et al., 1991).

\begin{tabular}{|c|c|}
\hline Categoria & $\mathrm{kg} / \mathrm{m}^{2}$ \\
\hline desnutrido & $\leq 18,49$ \\
\hline “baixo peso” & $18,50-19,99$ \\
\hline normal & $20,00-24,9$ \\
\hline sobrepeso & $25,00-30,0$ \\
\hline obeso & $>30,00$ \\
\hline
\end{tabular}

O padrão estabelecido pela OMS em 1990 define a desnutrição energética crônica pelos valores de IM C <18,5. Entretanto, julga-se importante manter os mesmos pontos de corte do PNSN para permitir a comparabilidade de prevalências de determinados Índices de Massa Corporal entre São Paulo e o restante do país. Assim, a categoria “baixo peso" está dentro dos limites de normalidade.
Figura 1

Vale do Município de Cotia, Estado de São Paulo, com indicação das cinco áreas de estudo da pesquisa.

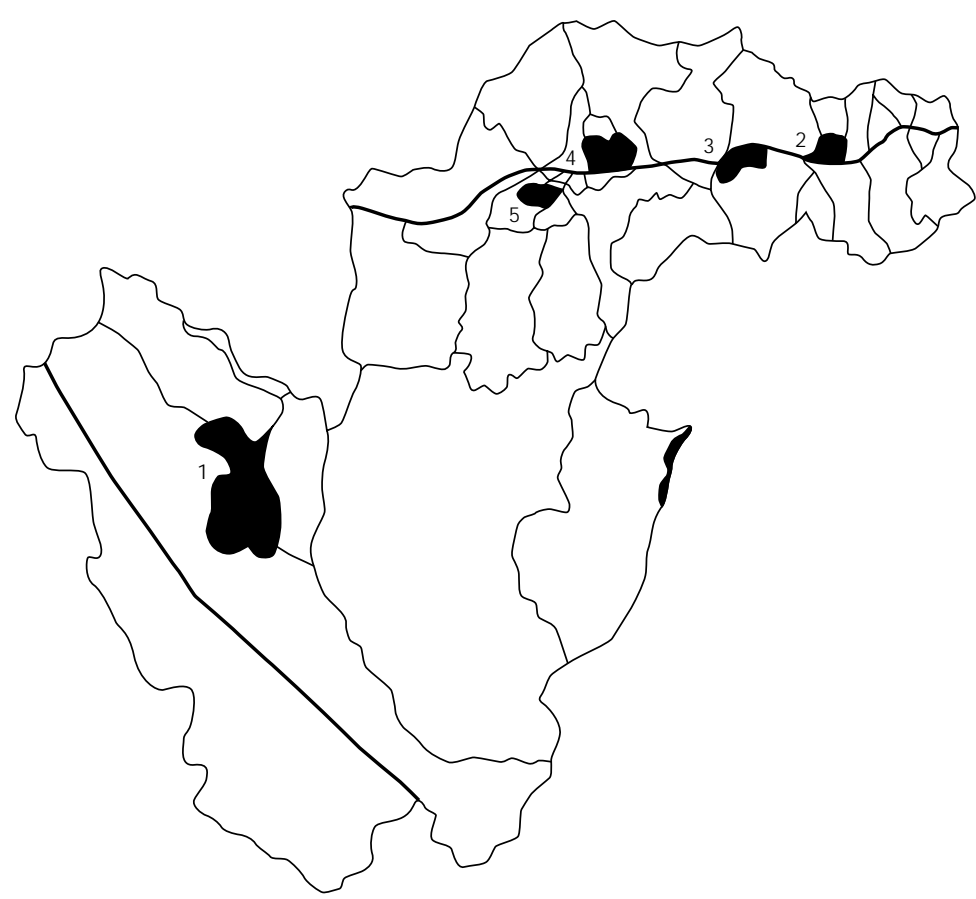

Área 1 - Planalto de Caucaia -

J ardim das O liveiras

Área 2 - Parque São J orge

Área 3 - J ardim Cláudio - Parque Alexandra J ardim Belizário

Área 4 - J ardim Coimbra -

J ardim Stella Maris - J ardim Cotia

Área 5 - J ardim Leonor

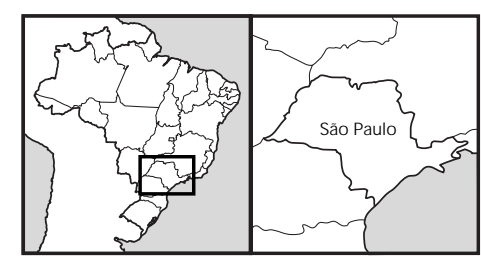

\section{A mostra}

Os domicílios foram as unidades amostrais de sorteio e as unidades elementares de observação foram os indivíduos com idade igual ou superior a 20 anos.

O tamanho da amostra foi estabelecido fixando-se um coeficiente de variação não superior a $5 \%$ para qualquer estimativa a ser estudada. Isto determinou para as situações de maior variabilidade, $p$ igual a 0,50, uma amostra de unidades elementares igual a 384. Ajustando esse número à correção devido ao possível efeito de conglomerado (deff $=1,5$ ), estabeleceu-se que o número mínimo de indivíduos da amostra deveria ser igual a 620. Pressupondo-se uma taxa de participação de 0,80 , o número seria igual a 775. Considerando-se o nú- 
mero médio de duas pessoas por domicílio, encontrado em um estudo piloto realizado na “área de estudo 1", concluiu-se que o número de domicílios sorteados deveria ser igual a 388. Por outro lado, por se tratar de uma pesquisa envolvendo inquéritos clínico-bioquímico e nutricional estendeu-se para 1000 o número de unidades elementares de observação envolvendo, assim, 500 domicílios.

No processo de amostragem foram utilizados mapas das quadras, das áreas de estudo selecionadas, indicando os lotes de cada uma, nome da rua e número do lote. No caso de um lote conter mais de um domicílio, estes seriam numerados de um até o total dos mesmos e um deles seria selecionado por sorteio.

Definiu-se uma amostra representativa para cada área de estudo e a amostra final foi o somatório das amostras de todas áreas, perfazendo a quantia de 1041 unidades elementares de observação.

Informações detalhadas sobre os inquéritos e medidas clínico, bioquímicas e alimentares realizadas encontram-se detalhadas em publicação a respeito da metodologia da pesquisa (Martins et al., 1993).

\section{Variáveis estudadas}

Idade e sexo: obtidos através de entrevista;

Peso: obtido através de pesagem do indivíduo, com roupas leves e sem sapatos, utilizando-se balança Filizola, com sensibilidade de $100 \mathrm{~g}$.

Altura: medida com fita métrica inelástica, com sensibilidade de $0,1 \mathrm{~cm}$, fixada em uma parede plana e com a ajuda de um esquadro.

\section{Estatística utilizada}

Para a elaboração do banco de dados foi usado o programa Epiinfo e a estatística utilizada na comparação de prevalências entre as diferentes categorias foi o método $\chi^{2}$.

\section{Resultados}

A Tabela 1 apresenta os percentis dos Índices de Massa Corporal (IMC), segundo idade e sexo. Verifica-se que nas mulheres, acima de 40 anos de idade, o sobrepeso já se faz presente no P50 da população, situação encontrada apenas no P75 entre os homens. Ainda no P75, obesidade aparece nas mulheres de 50 a 59 anos. Por outro lado, a desnutrição ocorre no percentil 5 dos idosos e na faixa etária de 40-49 anos entre os homens e de 20-39 anos entre as mulheres.
A Tabela 2 mostra a distribuição das prevalências de desnutrição, "baixo peso", sobrepeso e obesidade, em homens e mulheres. Verificase entre as mulheres de até 39 anos, 8,5\% de desnutrição e 9,6 \% de "baixo-peso". Nas idosas, a prevalência é de $8,1 \%$. Na população feminina a prevalência de "baixo peso" é de 7,1\% e de desnutrição $6,2 \%$. Por sua vez, entre os homens, varia entre 3,4\%, para a faixa etária de 20-39 anos e 7,3\%, para a de 60-88 anos. O “baixo peso" (19,5\%) atinge principalmente os idosos. Na população masculina, 3,9\% correspondem à prevalência de desnutrição e $8,3 \%$ à de "baixo peso". Na amostra como um todo as prevalências de "baixo peso" e desnutrição são de $8,3 \%$ e $5,3 \%$, respectivamente.

No que tange ao sobrepeso, é al ta a prevalência na população: $26,6 \%$, 38\%, 35,0\% e 37,8\%, respectivamente, para as faixas etárias de 20 39 anos, 40-49 anos, 50-59 anos e acima de 60 anos. Nos homens é discreta a variação de prevalências entre os grupos etários: 27,5\% (20-39 anos), 30,9\% (40-49 anos), 31,5\% (50-59 anos) e $31,4 \%$ ( 60 anos e mais). Em relação às mulheres constata-se que ao atingirem a faixa dos 40 anos a prevalência aumenta sensivelmente, de $25,8 \%$ para $43,6 \%$, permanecendo alta nas décadas posteriores ( $p \varangle 0,001)$. Até essa idade as prevalências entre homens e mulheres são próximas. Após esse período, há um aumento expressivo do sobrepeso e obesidade nas mulheres e os diferenciais entre os sexos passam a ser estatisticamente significantes ( $p \varangle 0,001)$.

A obesidade, na população amostrada, é verificada com perfil de prevalência diferente ao do sobrepeso: aumento brusco de 5,5 a $13,1 \%$, entre os períodos de $20-39$ e 40-49 anos, atingindo as maiores taxas aos 50-59 (21,3\%), voltando a diminuir após os 60 .

A Tabela 3 trata da proporção de homens e mulheres com Índice de Massa Corporal menor do que $20 \mathrm{~kg} / \mathrm{m}^{2}$, segundo estrato social, padronizadas em relação à idade, pelo método direto. As porcentagens situam-se entre, mais ou menos, 15 e $18 \%$. Entre as mulheres as prevalências encontram-se entre 11,8 e $26,5 \%$ nos estratos de mais alto e de mais baixo poder aquisitivo, respectivamente. No que diz respeito aos homens os percentuais situam-se entre $11,8 \%$ (classe II) e 20,0\% (classe IV).

A Tabela 4 apresenta as prevalências de Índice Massa Corporal maior do que $25 \mathrm{~kg} / \mathrm{m}^{2}$ em homens e mulheres, nos diferentes estratos sociais. Constata-se, entre os homens, percentuais de cerca de 43, 51, 34 e $30 \%$, nos estratos I , II, III e IV, respectivamente. Nas mulheres, verifica-se tendência inversa em relação aos estratos sociais: nos estratos II, III e IV são mais 
Tabela 1

Distribuição do índice de massa corporal em percentis de acordo com o sexo e idade. Município de Cotia 1990-91.

\begin{tabular}{crrrrrrrr}
\hline & $\mathrm{n}$ & \multicolumn{7}{c}{ percentil } \\
& & 5 & 10 & 25 & 50 & 75 & 90 & 95 \\
\hline $\begin{array}{c}\text { Homens } \\
20-39\end{array}$ & 236 & 18,73 & 19,31 & 21,13 & 23,20 & 25,46 & 28,32 & 29,05 \\
$40-49$ & 97 & 18,17 & 19,10 & 21,51 & 24,15 & 26,78 & 29,70 & 32,17 \\
$50-59$ & 54 & 19,96 & 20,75 & 23,24 & 24,79 & 27,70 & 30,18 & 32,87 \\
$60-88$ & 41 & 18,24 & 18,80 & 20,58 & 24,50 & 26,91 & 30,06 & 31,62 \\
$20-88$ & 428 & 18,73 & 19,34 & 21,34 & 23,69 & 26,37 & 28,71 & 30,12 \\
Mulheres & & & & & & & & \\
$20-39$ & 364 & 18,18 & 18,97 & 21,05 & 23,53 & 26,37 & 29,24 & 30,89 \\
$40-49$ & 124 & 20,61 & 21,78 & 23,42 & 26,33 & 28,91 & 31,70 & 35,16 \\
$50-59$ & 63 & 20,70 & 21,56 & 24,05 & 27,41 & 30,30 & 32,89 & 35,70 \\
$60-88$ & 62 & 17,35 & 19,63 & 21,98 & 25,68 & 29,44 & 34,68 & 37,39 \\
Total & & & & & & & & \\
$20-88$ & 613 & 18,43 & 19,58 & 21,76 & 24,53 & 27,59 & 30,84 & 33,91 \\
\hline
\end{tabular}

Tabela 2

Distribuição de estados nutricionais, segundo idade e sexo. Município de Cotia, 1990-1991.

\begin{tabular}{|c|c|c|c|c|c|c|c|c|c|c|c|}
\hline & \multirow[t]{2}{*}{$\mathrm{N}$} & \multicolumn{2}{|c|}{$\begin{array}{l}\text { desnutrido } \\
\leq 18,49\end{array}$} & \multicolumn{2}{|c|}{$\begin{array}{l}\text { baixo peso } \\
18,50-19,99\end{array}$} & \multicolumn{2}{|c|}{$\begin{array}{c}\text { normal } \\
20,00-24,99\end{array}$} & \multicolumn{2}{|c|}{$\begin{array}{c}\text { sobrepeso } \\
25,00-30,00\end{array}$} & \multicolumn{2}{|c|}{$\begin{array}{l}\text { obesidade } \\
>30,00\end{array}$} \\
\hline & & $\mathrm{n}$ & $\%$ & $n$ & $\%$ & $n$ & $\%$ & $n$ & $\%$ & $n$ & $\%$ \\
\hline \multicolumn{12}{|l|}{ Total } \\
\hline $20-39$ & 600 & 39 & 6,5 & 55 & 9,2 & 314 & 52,3 & 160 & $26,7 \mathrm{a}$ & 32 & $5,3 \mathrm{a} 1$ \\
\hline $40-49$ & 221 & 7 & 3,2 & 12 & 5,4 & 89 & 40,3 & 84 & $38,0 \mathrm{~b}$ & 29 & $13,1 \mathrm{~b} 1$ \\
\hline $50-59$ & 117 & 1 & 0,9 & 4 & 3,4 & 46 & 39,3 & 42 & $35,9 c$ & 24 & $20,5 \mathrm{cl}$ \\
\hline $60-88$ & 103 & 8 & 7,8 & 11 & 10,7 & 28 & 29,5 & 39 & $37,8 d$ & 17 & $16,5 \mathrm{~d} 1$ \\
\hline $20-88$ & 1041 & 55 & 5,3 & 82 & 8,3 & 477 & 46,8 & 325 & 31,2 & 102 & 9,8 \\
\hline \multicolumn{12}{|c|}{ Mulheres } \\
\hline $20-39$ & 364 & 31 & 8,5 & 35 & 9,6 & 178 & 48,0 & 94 & $25,8 \mathrm{a} 2$ & 26 & 7,1a3 \\
\hline $40-49$ & 124 & 1 & 0,8 & 4 & 3,2 & 45 & 36,5 & 54 & $43,6 \mathrm{~b} 2$ & 20 & $16,1^{b 3}$ \\
\hline $50-59$ & 63 & 1 & 2,5 & 1 & 1,6 & 19 & 30,2 & 24 & $38,1 \mathrm{c} 2$ & 18 & $28,5 \mathrm{c} 3$ \\
\hline $60-88$ & 62 & 5 & 8,1 & 3 & 4,8 & 16 & 25,8 & 25 & $40,3 \mathrm{~d} 2$ & 13 & $20,9 \mathrm{~d} 3$ \\
\hline $20-88$ & 613 & 38 & 6,2 & 43 & 7,1 & 258 & 42,1 & 197 & 32,1 & 77 & 12,6 \\
\hline \multicolumn{12}{|l|}{ Homens } \\
\hline $20-39$ & 236 & 8 & 3,4 & 20 & 8,5 & 136 & 57,6 & 65 & 27,5 & 6 & 2,5 \\
\hline $40-49$ & 97 & 6 & 6,2 & 8 & 8,3 & 44 & 45,3 & 30 & 30,9 & 9 & 9,3 \\
\hline $50-59$ & 54 & 0 & 0,0 & 3 & 5,6 & 27 & 50,0 & 17 & 31,5 & 6 & 11,1 \\
\hline $60-88$ & 41 & 3 & 7,3 & 8 & 19,5 & 12 & 29,2 & 14 & 34,1 & 4 & 9,8 \\
\hline $20-88$ & 428 & 17 & 3,9 & 39 & 9,1 & 219 & 51,2 & 126 & 29,4 & 25 & 5,8 \\
\hline
\end{tabular}

a.b $<0,025$

a1.b1.c1.d1 - p <0,005; a1.b1- $p<0,01 ; a 1 . c 1-p<0,005 ; a 1 . d 1-p<0,005$

a2.b2.c2.d2 - p <0,03; a2.b2-p<0,005

a3.b3.c3.d3 - p<0,005; a3.b3-p<0,05; a3.c3.-p<0,005; a3.d3-p<0,03 
Tabela 3

Prevalência de baixo peso em homens e mulheres segundo classes sociais. Município de Cotia, 1990-1991.

\begin{tabular}{|c|c|c|c|c|c|c|}
\hline \multirow[t]{2}{*}{ Estratos } & \multicolumn{3}{|c|}{ homens } & \multicolumn{3}{|c|}{ mulheres } \\
\hline & $\mathrm{n}$ & $\mathrm{c}^{*}$ & $\%$ pd** & $\mathrm{n} *$ & $\mathrm{C}^{* *}$ & $\% p d$ \\
\hline I & 35 & 3 & 16,1 & 43 & 5 & 11,8 \\
\hline II & 90 & 9 & 14,6 & 92 & 12 & 17,1 \\
\hline III & 219 & 29 & 13,8 & 336 & 45 & 17,6 \\
\hline V & 84 & 15 & 18,1 & 138 & 19 & 26,5 \\
\hline Total & 428 & 56 & 13,1 & 613 & 81 & 13,2 \\
\hline
\end{tabular}

* casos

** padronizada de acordo com idade

Tabela 4

Prevalência de sobrepeso e obesidade em homens e mulheres, segundo classes sociais. Município de Cotia, 1990-1991.

\begin{tabular}{|c|c|c|c|c|c|c|}
\hline \multirow[t]{2}{*}{ Estratos } & \multicolumn{3}{|c|}{ homens } & \multicolumn{3}{|c|}{ mulheres } \\
\hline & $\mathrm{n}$ & $\mathrm{C}$ & $\% p d$ & $n$ & $c$ & $\% p d$ \\
\hline I & 35 & 15 & 43,3 & 43 & 7 & 11,8 \\
\hline II & 90 & 44 & 50,7 & 96 & 49 & 61,5 \\
\hline III & 219 & 70 & 34,5 & 336 & 155 & 54,7 \\
\hline IV & 84 & 24 & 29,5 & 138 & 63 & 45,6 \\
\hline Total & 428 & 153 & 35,7 & 613 & 274 & 44,7 \\
\hline II.III $p<0,05$ & & \multicolumn{2}{|c|}{ I.II.III.IVp $<0,005$} & \multicolumn{3}{|c|}{ II.III $p<0,005$} \\
\hline \multirow[t]{2}{*}{ II.IV $p<0,05$} & & \multicolumn{2}{|c|}{ I.II $p<0,005$} & \multicolumn{3}{|c|}{ II.IV $p<0,005$} \\
\hline & \multicolumn{3}{|c|}{ I.IV $p<0,005$} & & & \\
\hline
\end{tabular}

altas do que no I. Tem-se a menor prevalência no estrato I e a maior no II ( $p<0,005)$. Entretanto, os estratos III e IV também apresentam altos percentuais de obesidade, aproximadamente 55 e $46 \%$, respectivamente. No cômputo geral, as mulheres apresentaram prevalências, estatisticamente significantes, mais altas que os homens $(p \triangleleft 0,05)$.

\section{Discussão}

Subnutrição não ultrapassa 5\% da população, atingida por alta prevalência de excesso de peso. Estes dados contradizem, aparentemente, o estudo de Cervato et al. (1995), que demonstra consumo de energia abaixo das recomendações em quase $50 \%$, desta amostra. Essa disparidade conduz à questão sobre as avaliações de consumo energético em estudos epidemiológicos, que são feitas através de tabelas de recomendações estimadas para a média das necessidades observadas de classes de indivíduos saudáveis. Com isso, as necessidades reais de parte da população podem estar superestimadas. Assim, espera-se que os comitês, no futuro, se preocupem com a construção de tabelas dirigidas à adequação de consumo para estudos populacionais. Por outro lado, existem mecanismos adaptativos para a economia de energia adquiridos em situações de desnutrição pregressa e a obesidade poderia ser resultante tanto dessa adaptação quanto de um consumo excessivo de energia. Ainda nesta população, Velásquez-Meléndez et al. (1998), encontra associação entre baixa estatura e obesidade, principalmente em mulheres. Outro determinante da obesidade é o sedentarismo, encontrado em $37 \%$ da população. Abrange quase $50 \%$ dos homens dos estratos de maior poder aquisitivo; os demais variam entre $36 \%$ (III) e 40\% (II eIV). Entretanto, no que tange às mulheres, menos de $20 \%$ do estrato I apresenta vida sedentária ao contrário das demais, cujas prevalências variam, aproximadamente, de 31 a 40\% (Martins et al., 1995).

Os maiores percentuais de obesidade e sedentarismo foram encontrados no estrato II , no qual está adstrita a classe média pouco ilustrada, formada por pequenos proprietários, geralmente ligados ao comércio.

Por outro lado, os padrões alimentares delineiam-se na população amostrada como fator de risco importante da obesidade. Em trabalho anterior (Martins et al., 1994), onde são apresentados o consumo de nutrientes energéticos nos estratos sociais, registra-se, entre os homens pertencentes ao estrato II, maior proporção de consumo e energia e de nutrientes. Nas dietas do estrato de maior nível sócio-econômico, prevalece o consumo de proteínas de origem animal, de forma mais moderada o de gorduras e em menor proporção o de carboidratos. Entre as mulheres, o consumo de proteínas é maior nos estratos I e II, verificando-se um nítido aumento de dietas ricas em carboidratos nos estratos de menor poder aquisitivo. O consumo relativo de lipídios, principalmente de origem vegetal, é alto em todos os estratos. O estudo realizado por Matos (1997) agrega outras informações sobre a aterogenicidade da dieta dessa população demonstrando, em geral, baixo consumo de fibras. Assim, nos grupamentos mais pobres encontra-se uma alimentação à base de carboidratos e gorduras, altamente indutoras da obesidade.

A Área M etropolitana de São Paulo (AMSP) situa-se em um contexto histórico que sofreu mudanças profundas nos padrões alimentares nas últimas décadas. $\mathrm{O}$ estudo de Monteiro et al. (1995), com dados de três inquéritos ali- 
mentares, realizados entre 1961 e 1988, constata aumento no consumo de gordura de origem vegetal, carne, leite e derivados. Aponta ainda, aumento expressivo na proporção de gorduras em relação às calorias totais da dieta. Demonstra, também, que na AMSP essa proporção sempre foi alta, ultrapassando, em 1988, as recomendações estabelecidas. Nesse mesmo período, observou-se na população brasileira aumento expressivo da obesidade quando se confrontam os dados do ENDEF e PNSN (Coitinho et al., 1991).

Por outro lado, comparando-se a população do presente estudo com a da PNSN, verificam-se prevalências de IMC $<20$ ligeiramente menores na AMSP do que no Brasil como um todo, $13,6 \%$ e $15,9 \%$, respectivamente. Para os homens a prevalência é de $15,4 \%$ (Brasil) e 13,0\% (AMSP); para as mulheres, 16,5\% (Brasil) contra 13,4\% (AMSP). Constata-se tanto no estudo da AMSP como no da PNSN tendência similar na distribuição das prevalências de desnutrição e IMC < 20 kg/m2: apresentam-se mais altas entre os mais jovens e nos idosos.

Em relação às prevalências de sobrepeso e obesidade, destacam-se os seguintes pontos: os percentuais de sobrepeso e obesidade atingem a $35,9 \%$ e $44,7 \%$ (AM SP) contra $28,0 \%$ e $38 \%$ (PNSN) dos homens e mulheres, respectivamente. Em ambos os estudos, o excesso de peso é observado entre as mulheres, principalmente após os 40 anos de idade. As prevalências na AMSP para ambos os sexos são mais altas, variando de $40-44 \%$ contra $34-36 \%$ (PNSN). Verifica-se ainda que na AMSP as taxas de prevalências são ainda mais el evadas do que as encontradas para a Região Sul Brasil da PNSN.

Por outro lado, os estados nutricionais, segundo nível sócio-econômico, apresentam as mesmas tendências na AMSP e PNSN (área urbana): entre as mulheres até 40 anos de idade IMC < 20 é maior nas classes de menor nível sócio-econômico; o excesso de peso guarda relação direta com o estrato social entre os homens e inversa entre as mulheres.

Assim, nos estratos sociais que compõem a população deste estudo, constata-se alta prevalência de sobrepeso e obesidade. Possivelmente a vida sedentária e uma alimentação aterogênica se apresentem como os principais fatores de risco na população.

\section{Agradecimentos}

À Fundação de Amparo à Pesquisa do Estado de São Paulo (Fapesp), processo no 91\0820-7 e ao Conselho Nacional de Desenvolvimento Científico e Tecnológico (CNPq), processo oo 410427 89-6, pelo financiamento deste trabalho.

\section{Referências}

BRAY, G. L., 1985. Obesity definition, diagnosis and disadevantages. Medical Journal of Australia, 142: 52-58.

BREILH, J. \& GRANDA, E., 1980. Investigación de Salud en Sociedad: Guia Pedagógico Sobre un Nuevo Enfoque del Método Epidemiológico. Quito: Ediciones C.E.A.S.

CASTELLS, M., 1971. Que és sociologia urbana? In: Problemas de Investigación en Sociologia Urbana (M. Castells, ed.), pp. 15-45, Madrid: Siglo Vienteuno de España. 
CERVATO, A. M.; MAZZILLI, R. N.; MARTINS, I. S. \& MARUCCI, M. F. N., 1995. Dieta habitual e fatores de risco para doenças cardiovasculares. Revista deSaúdePública, 31:227-231.

COITINHO, D. C.; LEÃO, M. M.; RECINE, E. \& SICHIERI, R., 1991. Condições Nutricionais da População Brasileira: Adultos eldosos. Brasília: INAN (Instituto Nacional de Alimentação e Nutrição).

FERRO-LUZZI, A.; SETTE, S.; FRANKLIN, M. \& JAMES, W. P. T., 1992. A simplied approach to assessing adult chronic energy deficiency. European Journal of Clinical Nutrition, 46:173-186.

JAMES, W. P. T.; FERRO-LUZZI, A. \& WATERLOW, J. C., 1988. Definition of chronic energy deficiency in adults. Report of working party of the International Dietary Energy Consultive Group. European Journal of Clinical Nutrition, 42: 961-981.

LAURELL, A. C., 1982. La salud - enfermedad como proceso social. Cuadernos Médico Sociales, 19:720.

LOMBARDI, C.; BRONFMAN, M.; FACCHINI, L. A.; VICTORA, C. G.; BARROS, F. C.; BÉRIA, U. J. \& TEIXEIRA, A. M., 1988. Operacionalização do conceito de classes sociais em estudos epidemiológicos. Revista de Saúde Pública, 22:253-265.

MARTINS, I. S.; COELHO, L. T.; MAZZILLI, R. N.; SINGER, J. M.; SOUZA, C. U.; ANTONIETO Jr., A.; PASINI, U.; NIETO, R. A.; ÁLVAREZ, E. D. \& OKANI, E. T., 1993. Doenças cardiovasculares ateroscleróticas, dislipidemias, hipertensão, obesidade e diabetes melittus em população da região sudeste do Brasil: I. Metodologia da Pesquisa. Revista de SaúdePública, 27:250-261.

MARTINS, I. S.; MAZZILLI, R. N.; NIETO, R. A.; ALVAREZ, E. D.; OSHIRO, R.; MARUCCI, M. F. N. \& CASAJUS, M. I., 1994. Hábitos al imentares aterogênicos de grupos populacionais da em área metropolitana da região sudeste do Brasil. Revista de SaúdePública, 28:349-356.
MARTINS, I. S.; COELHO, L. T.; CASAJUS, M. I. \& OKANI, E. T., 1995. Smoking, consumption of alcohol and sedentary life style in population grouping and their relatinships with lipemic disorders. Revista de Saúde Pública, 29:38-45.

M ATOS, L. L., 1997. Consumo de Fibras Ali mentares em População Adulta da Região Metropolitana de São Paulo. Dissertação de Mestrado, São Paulo: Faculdade de Economia e Administração e Faculdade de Ciências Farmacêuticas da Universidade de São Paulo.

MONDINI, L. \& MONTEIRO, C. A., 1995. Mudanças no padrão de alimentação. In: Vel hos e Novos Males da Saúde no Brasil: A Evolução do País e de suas Doenças (C. A. Monteiro, org.), pp. 79-89, São Paulo: Hucitec

MONTEIRO, C. A.; MONDINI, L.; SOUZA, A. L. M. \& POPKIN, B. M., 1995. Da desnutrição para a obesidade: a transição nutricional no Brasil. In: VeIhos e Novos Males da Saúde no Brasil: a Evolução do País e de suas Doenças (C. A. Monteiro, org.), pp. 248-255, São Paulo: Hucitec.

OMS (Organización Mundial de la Salud), 1990. Die ta, Nutrición y Prevención de Enfermedades Crónicas. Informes Técnicos, 797. Ginebra: OMS.

SINGER, P. I., 1981. Dominação e Desi gualdade: Estrutura deClasses e Distribuição de Renda no Brasil. Rio de Janeiro: Paz eTerra.

VELASQUEZ-M ELENDEZ, G.; M ARTINS, I. S.; CERVATO, A. M.; FORNES, N. S.; MARUCCI, M. F. N. \& COELHO, L. T., 1998. Relationship between central adiposity, body mass index, food intake, blood lipids and height in a Brazilian population. (in press). 